\title{
SPECIAL TOPIC ON MULTISCALE MODELING OF GRANULAR MEDIA: A TRIBUTE TO PROF. DOMINIQUE JEULIN
}

\author{
JEAN SERRA ${ }^{1}$ AND FRANÇOIS WILLOT ${ }^{\bowtie, 2}$ \\ ${ }^{1}$ Laboratoire d'Informatique Gaspard-Monge, ESIEE, Paris-Est University, France, ${ }^{2}$ Centre for Materials, PSL \\ Research University, Mines ParisTech, France \\ e-mail: jean.serra@esiee.fr, francois.willot@ensmp.fr
}

\begin{abstract}
A few words on the present special topic, devoted to the multiscale modeling of granular media, and published in honor of Prof. Dominique Jeulin's enduring contribution to the wide field of image analysis, random structures and material science.
\end{abstract}

Keywords: Image analysis; Stereology; Integral Geometry; Homogenization.

It has been a pleasure to assist Marko Kreft in editing and publishing the present special topic honoring Dominique Jeulin's contribution to science, research and teaching. This special topic, together with a special issue of International Journal of Solids and Structures (Elsevier) and a recentlypublished book (Willot \& Forest, 2018) stem from the international workshop ${ }^{1}$ organized in the island of Oléron, France, on June 17-22, 2018. Included in the following special topic are four manuscripts: Some dense random packings generated by the dead leaves model by D. Jeulin, Eikonal-based models of random tessellations by B. Figliuzzi, Tortuosimetric operator for complex porous media characterization, by J. Chaniot, M. Moreaud, L. Sorbier, T. Fournel \& J.-M. Becker and Region homogeneity in the logarithmic image processing framework: application to region growing algorithms by $\mathrm{G}$. Noyel \& $\mathrm{M}$. Jourlin. We would like to thank the researchers who conscientiously reviewed articles that were submitted to us, suggesting improvements. and also to thank the authors of a very good manuscript we had to decline, in accordance with the reports of the reviewers and the journal's constraints.

We are indebted to many colleagues and friends who have provided support for the organization of the workshop leading to the present special topic. We are grateful in particular to Samuel Forest, Anne-Marie De Castro, Sandra Rodrigues, Hugues Talbot, Lucie Kubínová, Aleš Kladnik, Marko Kreft, Félix Latourte, Jesús Angulo, Alain Fanget, Christian Lantuéjoul, François Mudry, Hervé Trumel, Michel Bilodeau and Jérôme Crépin. We are also grateful to the École des Mines, the Centre for Mathematical Morphology and the Centre for Materials P. M. Fourt, the Armines research association, the International
Society for Stereology \& Image Analysis, Électricité de France and the association Mécamat for their financial support.

The present editorial page is a good opportunity to write a few words on D. Jeulin's enduring contribution to the field of random structures, microstructure characterization and homogenization. We do not pretend to develop a biography of D. Jeulin in the present editorial preface, but rather to emphasize certain key events in his professional career where new orientations have been taken, or, to quote Soljenitsyne, where "nodes" have appeared.

In 1972, Dominique Jeulin was spending one year at the University of Lomonossov in Moscow (see photograph, Fig. 1), at the chair of engineering geology, under the guidance of Prof. Evguenei Nikolaievitch Kolomenski. This was, after graduating from the École des Mines of Nancy, Dominique's first work. Russian engineers were trying to build dams on the north side of Caucasus, and geologists had been studying resistance to crushing of dolerite and basalt rocks, in conjunction with their petrographic structure. In this faraway time, D. Jeulin's first steps in research were already aimed at elucidating the links between the geometry of heterogeneous media and physical properties. Once a track is followed, one rarely escapes it.

The approach of the time, based almost entirely on statistics, was rather scanty. Mechanical and physical responses were matched with petrographic measurements, and in effect to openings in two dimensions, following J. Pierre Benzécri's popular method in these days. No probabilistic model, or partial derivative equations, or numerical simulation, which are much more powerful tools than correlations,

\footnotetext{
${ }^{1}$ http://www.cmm.mines-paristech.fr/ willot/PMRM/ (accessed March 22, 2019).
} 
were used. Furthermore, the human factor, which produces materials, while geology only observes what nature has devised, was not taken into account in these Muscovite times. Time was not yet mastered.

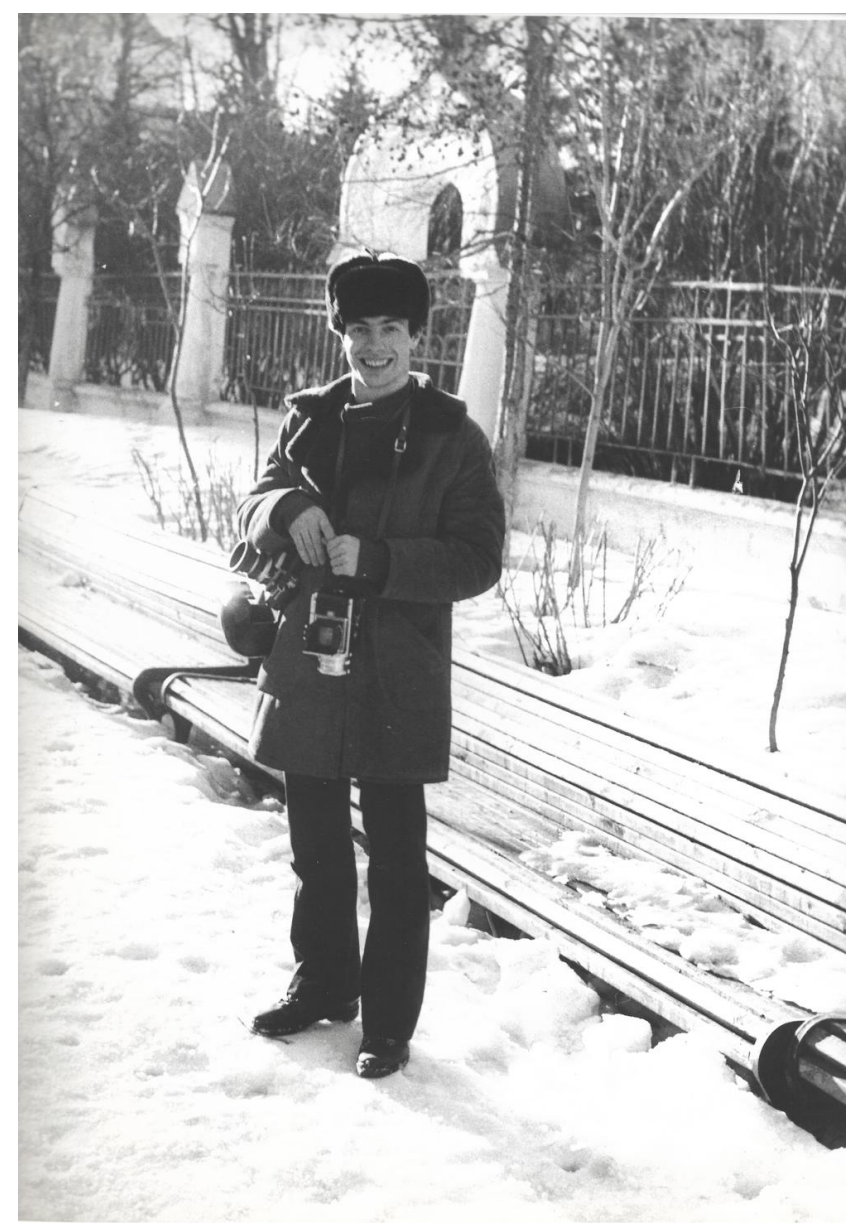

Fig. 1. D. Jeulin, Zagorsk, Russia, 1973.

This leads us to the second node, namely the IRSID, the Institute for Research in Steel Industry. It is well known that steel comes from iron oxides, and that blast furnaces remove oxygen from minerals and agglomerates. But geometrically, how does that happen? How does the sequence:

$$
\mathrm{Fe}_{2} \mathrm{O}_{3} \rightarrow \mathrm{Fe}_{3} \mathrm{O}_{4} \rightarrow \mathrm{FeO} \rightarrow \mathrm{Fe}
$$

comes into play? An agglomerate pellet is a porous medium and the reducing gas, which circulates through the pores, attacks the solid phase from its edges. Hence, the structure of the porous network must be tailored in order for the pellet not to crash too fast when it enters the blast furnace. But what one must do, and how to do it? This is the knotty question that IRSID asked Dominique when he came back from Moscow. Dominique provided answers in his doctoral thesis in 1979.

As years went by, IRSID, with its very specific problems, offered Dominique a rather limited horizon.
In 1985, Dominique decided to take a great step, and entered the industrial world at Thomson in Dijon. The industrial adventure did not exceed one year, and this third node appears to us, looking back now, as a brief parenthesis between the two major scientific adventures of Dominique.

The École des Mines welcomed him in Fontainebleau, first in the Center of Geostatistics, and a few years later in the Center for Mathematical Morphology. A new adventure took place.

D. Jeulin's ambition was to create a team acting in two domains, as his approach, like Janus, combined two different fields, random set geometry and the physics of heterogeneous media. And he succeeded! Fontainebleau is not far from Corbeil's Center for Materials, and the École des Mines appreciated that someone would dare to follow transverse paths between different worlds.

This approach was very fruitful. During thirty years, Dominique has taken over questions of statistical fracture, homogenization of composite materials, three-dimensional simulation of structures, transport of particles and nanoparticles, microstructure evolution under various constraints, etc. There are surely many other, but most importantly, he created and developed an outstanding arsenal of methods and models.

Dominique Jeulin modeled, simulated, calculated and predicted the properties of many heterogeneous materials relying on the tools of mathematical morphology and thinking in terms of probabilistic models. D. Jeulin's multiple approaches were not limited to the methods of mathematical morphology and random sets as developed by G. Matheron. The theory of percolation or gas network, to name a few, were not born in Fontainebleau, but he knew how to master and magnify them.

The scientific world, like all inventors and creators, is inhabited by two kinds of very different minds, those who have several ideas, and those who have one. In art, this separates Chopin from Picasso. Dominique is the man of an idea, as was Georges Matheron.

A central idea is like the trunk of a tree. When it grows up, branches develop in all directions, all linked to the trunk. Some of these branches are gathered in this issue, as a tribute to the tree who irrigated them.

\section{REFERENCES}

Willot F, Forest S (eds). Physics et Mechanics of Random Media: from Morphology to Material Properties. Presses des Mines, Paris, 2018. 